\title{
Measles molecular epidemiology: What does it tell us and why is it important?
}

\author{
Hiebert $\mathrm{J}^{1 *}$ and Severini $A^{1}$ \\ 1 National Microbiology Laboratory, Public Health Agency of Canada, Winnipeg, MB \\ * Correspondence to: Joanne.Hiebert@phac-aspc.gc.ca
}

\begin{abstract}
Background: Measles molecular epidemiology was a key component of the verification of elimination of indigenous measles in Canada and is an invaluable tool during public health investigations, both to establish whether connections exist between concurrent measles cases and to indicate possible sources of importations. There are 24 distinct genotypes however the genotype is usually not sufficient to describe the complex molecular epidemiology of measles cases. The exact genetic sequence of the last 450 nucleotides of the nucleoprotein $(\mathrm{N})$ gene $(\mathrm{N}-450)$ is used. The measles genome mutates very slowly and so cases within the same chain of transmission usually have identical N-450 sequences. In Canada, the National Microbiology Laboratory (NML) sequences the N450 and deposits it into the WHO measles sequence database, MeaNS. This database can be used to identify other geographic regions where the measles sequence was detected, supporting or excluding connections. For commonly detected N-450 sequences, MeaNS designates a "sequence variant." Sequence variants are used as the defining characteristic of measles cases with identical sequences and this designation is fundamental to the description of measles molecular epidemiology both locally and globally. As progress is made towards global measles eradication, its genetic diversity decreases and distinct importations of measles from a single reservoir can be difficult to distinguish using current methods. Extending sequencing methods beyond the $\mathrm{N}-450$ is required. While sequencing the entire hemagglutinin $(\mathrm{H})$ gene, which is currently done routinely at the NML, can be helpful, whole genome sequencing will be required for effective molecular surveillance to monitor the sustained elimination of measles in Canada.insert text here.
\end{abstract}

\section{Introduction}

Canada has eliminated indigenous measles, an achievement documented in a report submitted to the Pan American Health Organization (PAHO)/World Health Organization (WHO) (1). The last endemic measles case in Canada occurred in 1997, and measles was eliminated from the entire region of the Americas in 2002 (2). Measles molecular epidemiology was a key component in verifying the elimination of measles by demonstrating the absence of an endemic genotype and will continue to be critical in the monitoring of measles elimination. Furthermore, measles molecular epidemiology is an invaluable tool during public health investigations, both to establish whether connections exist between concurrent measles cases or outbreaks and to indicate possible sources of importations. This article provides information with which to understand measles genotyping data, to demonstrate both the utility of and need for measles molecular epidemiology in Canada and to provide a discussion of the future directions in an elimination setting.

\section{Measles genotyping 101}

An important aspect of disease surveillance is the ability to differentiate lineages, types or variants of a pathogen. This process is generally referred to as typing and, historically, pathogens of the same genus or species would be typed by their phenotypic characteristics, such as biochemical markers (e.g. presence or absence of toxins) or serological markers (the types of antigens present on the pathogen). This is largely the process used for enteric bacteria (Salmonella, E. coli, etc.) and influenza viruses for example. However, for a number of pathogens, including measles, there are insufficient phenotypic differences to employ this method of typing. The advent of DNA sequencing technology, the process of determining the order and identity of 
nucleotides within a DNA molecule, has allowed the identification of distinguishing regions within genomes that can be used for differentiating lineages. This is the process of genotyping.

The WHO has identified 24 phylogenetically distinct measles genotypes, designated A, B1, B2, B3, C1, C2, D1, D2, D3, D4, D5, D6, D7, D8, D9, D10, D11, E, F, G1, G2, G3, H1 and H2, in which the letters identify the main clade and the numbers the subclades (3). Genotypes are groupings of genetically related sequences with inherent variability, and the genotype designation is usually not sufficient to describe the complex molecular epidemiology of measles cases. Therefore the WHO recommends using the exact sequence (or "variant") of the last 450 nucleotides of the nucleoprotein $(\mathrm{N})$ gene (termed the "N-450"), at a minimum, for molecular epidemiology and the entire sequence of the hemagglutinin $(\mathrm{H})$ gene $(1854 \mathrm{nt})$ for additional information $(3,4)$. Since the measles genome mutates very slowly, usually cases in the same outbreak or chain of transmission carry identical N-450 sequences, and differences, even of one nucleotide, are usually enough to exclude direct transmission between two cases.

The WHO maintains a measles sequence database, MeaNS (5) (http://www.whomeasles.org/Public/Web_Front/sequence.php), to which members of the WHO Global Measles Laboratory Network (LabNet) submit sequences ( $\mathrm{N}-450$ and $\mathrm{H}$ gene) from measles cases. LabNet members are also able to compare the $\mathrm{N}-450$ sequences of their cases to sequences deposited by other members in order to identify other geographic regions where the measles sequence was detected. This information can provide laboratory evidence supporting or excluding connections between imported cases and their countries of export.

For commonly detected N-450 sequences, MeaNS designates a "sequence variant" (3). Examples of sequence variants are MVs/Manchester.GBR/10.09 (genotype D4) and MVs/Taunton.GBR/27.12 (genotype D8). Members of the LabNet interrogate the MeaNS database to determine to which sequence variant the sequence from their measles case is identical. The sequence variant becomes the defining characteristic of measles cases and outbreaks, and is used to easily identify matching measles sequences from other possibly connected cases (within an outbreak for example) or countries of interest.

In Canada, measles genotyping is performed at the National Microbiology Laboratory (NML), a WHO regional measles and rubella reference laboratory. Provincial laboratories are advised to submit all their positive and suspect measles specimens (nasopharyngeal swabs and/or urine) to the NML for genotyping. While all measles cases should be genotyped, only those with adequate specimens can be, and are, genotyped. The NML returns the measles genotyping information, consisting of genotype, "sequence designation" and, where applicable, designation of the identical sequence variant, to the provinces. Sequence designations are the WHO standardized names of measles sequences obtained from individual measles cases. They serve to indicate both the place (city or province, or state and country) and time (by epidemiological week and year of rash onset or specimen collection) of the measles case $(3,4)$. For example MVs/Ontario.CAN/22.13 is a measles sequence from a case in Ontario, Canada, in the 22nd epidemiological week of 2013. The NML also reports to the WHO by depositing the sequences in MeaNS.

\section{Utility of and need for measles molecular epidemiology}

Canada underwent a substantial number of measles cases and outbreaks in 2013 , some of which occurred concurrently in different provinces (6). In some cases, identification of the measles genotype alone was sufficient to verify that concurrent outbreaks were indeed distinct (for example, early in 2013 genotype B3 measles cases occurred in Ontario shortly after the separate occurrence of genotype D8 cases in the same province [6]). In contrast, measles genotype D8 was detected throughout the year and in nearly every province that reported measles cases. On the surface this could be alarming for a country that has eliminated measles. Analysis of the sequence data served to demonstrate that the genotype D8 cases could be further characterised as four different sequence variants (6) and thus were not due to widespread circulation of one variant.

In addition to aggregate epidemiological analyses, measles genotyping can provide useful information during real-time investigation of measles cases. In 2013 measles was imported by two Canadian tourists who had independently travelled to the same resort in Mexico [6], another country that has eliminated endemic measles (2). Through genotyping and utilization of MeaNS it was possible to indicate the UK as a possible source of the 
virus (Kevin Brown, Public Health, England: personal communication, March 7, 2013), subsequently confirmed by the epidemiological investigation (6).

It should be noted that measles molecular epidemiology relies on the collection and submission of appropriate clinical specimens from all suspected measles cases (nasopharyngeal swab and/or urine; for details see (7) as well as the NML's Guide to Services, available at https://www.nml-Inm.gc.ca/guide2/index-eng.htm). In order to monitor the success of measles elimination programs, the WHO LabNet recommends genotyping at least $80 \%$ of all sporadic cases and outbreaks (i.e. genotyping at least one case from $\geq 80 \%$ of all outbreaks).

\section{Future directions}

The WHO has targeted measles for global eradication (8), and as progress is made the genetic diversity of measles viruses decreases. Often only one measles sequence variant is responsible for large sustained outbreaks, particularly in regions that have not eliminated measles. These outbreaks can be reservoirs for importations of measles into Canada. If there are multiple importations of the same variant, we are unable to distinguish separate importations using current measles genotyping methods. In 2011, the MVs/Manchester.GBR/10.09 sequence variant of genotype D4 was repeatedly imported into Quebec, British Columbia and Ontario and was linked to the large outbreak in Quebec (see [9] and unpublished data). The ability of molecular epidemiology to demonstrate the absence of endemic transmission was challenged. Extending genotyping methods beyond the $\mathrm{N}-450$ to collect additional sequence data for the purposes of finding distinctions between measles cases is therefore required. In 2013, the NML began routinely sequencing the $\mathrm{H}$ gene from every measles case. Distinct importations of the D8 strain from the Netherlands (identical to sequence variant MVs/Taunton.GBR/27.12) and unrelated outbreaks and cases of a single measles genotype B3 strain (identical to sequence variant MVi/Harare.ZWE/38.09), both of which were indistinguishable at the $\mathrm{N}-450$, could be differentiated using the $\mathrm{H}$ gene sequences (6). However, $\mathrm{H}$ gene sequencing is not always sufficient, and as a result whole genome sequencing will likely be required as we move forward toward global elimination.

\section{Conclusion}

Measles molecular epidemiology is an invaluable tool for tracking importations, linking cases and demonstrating the absence of sustained measles transmission. As the genetic diversity of measles virus decreases, extended genotyping and eventually whole genome sequencing will be required for effective molecular surveillance. Maintaining high-quality surveillance for measles cases, which includes genotyping (requiring the collection of appropriate specimens) is critical to monitoring the sustained elimination of measles in Canada.

\section{References}

(1) Public Health Agency of Canada. Elimination of measles, rubella and congenital rubella syndrome in Canada. Documentation and verification report. December 2011; http://www.phac-aspc.gc.ca/im/vpd-mev/measlesrougeole-mrer-eng.php.

(2) Castillo-Solorzano C C, Matus CR, Flannery B, Marsigli C, Tambini G, Andrus JK. The Americas: paving the road toward global measles eradication. J Infect Dis. 2011; 204(Suppl 1):S270-8.

(3) World Health Organization. Measles virus nomenclature update: 2012. Weekly Epidemiological Record. 2012; 9(87):73-80.

(4) World Health Organization. Standardization of the nomenclature for describing the genetic characteristics of wild-type measles viruses. Weekly Epidemiological Record. 1998; 73:265-272.

(5) Rota PA, Brown K, Mankertz A, Santibanez S, Shulga S, Muller CP, Hübschen JM, Siqueira M, Beirnes J, Ahmed H, Triki H, Al-Busaidy S, Dosseh A, Byabamazima C, Smit S, Akoua-Koffi C, Bwogi J, Bukenya H, Wairagkar N, Ramamurty N, Incomserb P, Pattamadilok S, Jee Y, Lim W, Xu W, Komase K, Takeda M, Tran T, Castillo-Solorzano C, Chenoweth P, Brown D, Mulders MN, Bellini WJ, Featherstone D. Global distribution of measles genotypes and measles molecular epidemiology. J Infect Dis. 2011; 204(Suppl 1), S514. 
(6) Public Health Agency of Canada. Measles surveillance in Canada: Trends for 2013; CCDR, 2014; Vol 4012:195.

(7) Measles and Rubella Elimination Working Group, Health Canada, Public Health Agency of Canada. Guidelines for the prevention and control of measles outbreaks in Canada. CCDR. 2013; 39:ACS-3. http://www.phac-aspc.gc.ca/publicat/ccdr-rmtc/13vol39/acs-dcc-3/index-eng.php\#appa.

(8) World Health Organization. Global eradication of measles: report by the Secretariat. Geneva, Switzerland: World Health Organization; 2010. http://apps.who.int/gb/ebwha/pdf_files/wha63/a63_18-en.pdf.

(9) De Serres G, Markowski F, Toth E, Landry M, Auger D, Mercier M, Bélanger P, Turmel B, Arruda H, Boulianne $\mathrm{N}$, Ward BJ, Skowronski DM. Largest measles epidemic in North America in a decade--Quebec, Canada, 2011: contribution of susceptibility, serendipity, and superspreading events. J Infect Dis. 2013; 207(6):9908.

\section{Acknowledgements}

The authors acknowledge Elizabeth McLachlan and Lillian Mendoza for critical review of the manuscript.

\section{Conflict of Interest}

No conflicts of interests to declare. 